\title{
Venetoclax rituximab en paciente con LLC con deleción 17p, reporte de un caso
}

\section{- Carmen Rosales}

Clínica del Country (Bogotá, D.C.)

Introducción: la LLC con deleción $17 p$ es de difícil manejo con las quimioterapias convencionales y medicamentos disponibles en la actualidad. En el mundo se dispone de un inhibidor selectivo de $B C L-2$, que ofrece en estos casos mejores resultados en supervivencia libre de progresión, especialmente al combinarlo con anti-CD20: rituximab.

Materiales y métodos: paciente masculino de 69 años se diagnostica en julio de 2014 LLC Rai 0 Binnet A, CMF con un 33\% de población linfoide B madura anormal con expresión de CD19, CD20, CD5, CD23, CD79B y BCL-2, no se detecta expresión de cadenas ligeras kappa, lambda. Del13q: 31,5\% y del17p: $64,5 \%$ ZAP 70 + 21\% inicialmente se dejó en observación. El 29 de marzo de 2016 se inició tratamiento con R-bendamustine, con progresión de la enfermedad a piel, confirmado con biopsia. Se inició ibrutinib el 3 de junio de 2016 hasta el 7 de diciembre de 2017, cuando presenta nuevamente linfocitosis hasta 111.750, se confirma del17p y IGVH no mutado; se empieza venetoclax rituximab el 9 de abril de 2018, alto riesgo de lisis tumoral. No evidencia complicaciones en escalonamiento de dosis. Hacia el día 48 presenta neutropenia y linfopenia, el venetoclax es suspendido en forma temporal. Por hipogammaglobulinemia se comienza IgG. Hay recuperación de leucopenia hacia la tercera semana de suspensión con reinicio del medicamento. Sin linfocitosis.

Resultados: debido al alto riesgo de lisis tumoral, se realizó seguimiento intrahospitalario estricto, pero no se presentó esta complicación; la neutropenia, como efecto secundario, tuvo recuperación sin complicaciones infecciosas. Es aún temprano para definir la respuesta, pero al menos se logró control de la linfocitosis.

Conclusiones: es la primera vez que se utiliza este esquema de tratamiento en Colombia; es un medicamento de fácil manejo, oral, ambulatorio y seguro, que abre oportunidades a pacientes con factores pronósticos adversos. 